\title{
A Novel Distributed Optical Fiber Sensing System Based on Parallel Computing
}

\author{
Lidong Lu, Yun Liang, Binglin Li, Jinghong Guo \\ China Electric Power Research Institute \\ Nanjing, 210003, China \\ lidong.lv@gmail.com
}

\begin{abstract}
A new Brillouin optical time domain reflectometry (BOTDR) based on parallel computing method to extract the spontaneous Brillouin scattering spectra is proposed. By use of parallel computing method, the speed of digital signal processing unit in the new BOTDR can be improved by more than 40 times, which benefits dynamic measurement of the temperature and/or strain along the fiber under test.
\end{abstract}

Keywords- sensing system; temperature monitoring; parallel computing; Brillouin spectra

\section{INTRODUCTION}

When laser light propagates in the optical fiber, spontaneous Brillouin scattering arises as the result of the interaction between the incident light and fiber core material. The Brillouin scattering spectrum is a Lorenz curve, and compared with the frequency of the laser light, the spontaneous Brillouin spectrum (Anti-Stokes component) is about $11 \mathrm{GHz}$ higher. The Brillouin spectrum is sensitive to the temperature and strain of the fiber, so it is a very useful way to monitoring the temperature and/or strain by measuring the change of Brillouin scattering spectra along the fiber, which is commonly called Brillouin frequency shift [1-3]. The Brillouin frequency shift linearly changes with temperature and strain, and the relationships can be expressed as

$$
\Delta v=\mathrm{C}_{\mathrm{T}} \Delta \mathrm{T}+\mathrm{C}_{\varepsilon} \Delta \varepsilon
$$

Where $\Delta v$ is Brillouin frequency shift, $\Delta T$ is the change of temperature, $\Delta \varepsilon$ is the change of strain, $C_{T}$ and $C_{\varepsilon}$ are temperature coefficient and strain coefficient respectively with $\mathrm{C}_{\mathrm{T}}=1.1 \mathrm{MHz} /{ }^{\circ} \mathrm{C}$ and $\mathrm{C}_{\mathrm{T}}=1 \mathrm{MHz} / 2 \mathrm{\mu} \varepsilon$.

For distributed optical fiber sensing, the location of each measurement points is crucial and optical time domain reflecting technology provides a perfect choice. It is a special optical radar technology, because the probe light pulse only propagates in the fiber under test (FUT) and the round trip time of the signal which is a litter longer than the pulse period corresponds to the location of scattering. Therefore, by measuring the spontaneous Brillouin spectra and their corresponding scattering time, the temperature and/or strain information can be accurately obtained, and the sensing system by this method is Brillouin optical time domain reflectometry (BOTDR). Each scattering location in the FUT returns a spontaneous Brillouin spectrum, and then by comparison with the original spectrum the Brillouin frequency shift is obtained. According to equation (1), the temperature and/or strain along the FUT can be extracted. Figure 1 shows a typical spontaneous Brillouin spectrum whose frequency is about $11 \mathrm{GHz}$ above the probe light frequency and when the temperature and/or strain changes, its frequency shifts accordingly as expressed in equation (1).

As light frequency is very high, it cannot be sensed by photo detector. To obtain the Brillouin spectrum, a local oscillator is adopted to mix with it in a coupler, then by coherent detection the Brillouin spectrum is converted to Brillouin electric spectrum that can be analyzed and processed in electric domain [4]. Usually, the Brillouin electric spectrum is in the range of several hundred megahertz, and then by a digital acquisition card with sampling rate of $1 \mathrm{GHz}$, the whole Brillouin spectrum can be captured. The Brillouin electric spectrum is shown in figure 2, and it can be expressed as,

$$
g(v)=\frac{C_{1}}{\left(v-v_{0}\right)^{2}+C_{2}}
$$

Where $v_{0}$ is the central frequency of Brillouin spectrum, $\mathrm{C}_{1}$ and $\mathrm{C}_{2}$ are two constant coefficients [3].

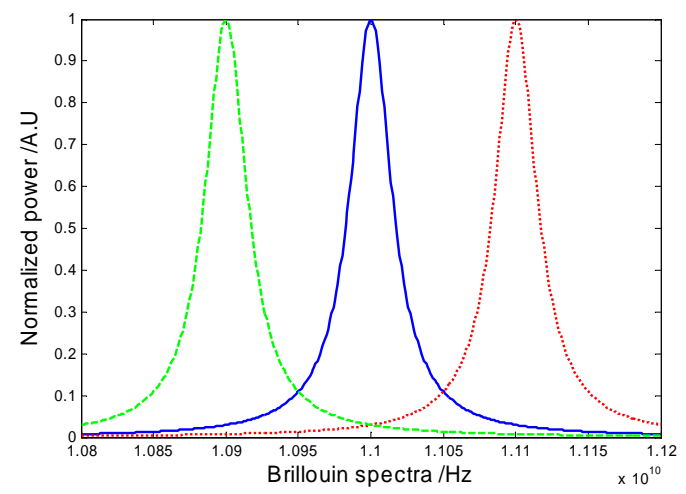

Figure 1. Spontaneous Brillouin spectrum in FUT.

As to extraction of the Brillouin spectrum, frequency sweeping method is used. First, a local oscillator (LO) is adopted to mix with the Brillouin spectrum, and then by coherent detection the Brillouin spectrum is transferred to electric spectrum output from a photo detector. Secondly a tunable microwave source (MWS) generates many discrete frequencies to sweep the Brillouin electric spectrum by heterodyne method, but in a probe pulse period only one frequency point in the Brillouin electric spectrum is swept. After a large number of frequencies are swept, the discrete 
Brillouin spectrum is obtained. At last, by data fitting algorithm the whole Brillouin spectrum can be obtained.

However, to get the whole Brillouin spectrum as shown in figure 2, tens of frequency pointes must be swept and when the number of frequency points that need to be swept increases, much longer time will be needed to obtain the whole Brillouin spectrum, so it greatly limits the dynamic measurement property of BOTDR. As to this defect existing in conventional BOTDR, we propose a new scheme that uses a high speed digital acquisition card (DAQ) to obtain the whole Brillouin electric spectrum and by parallel digital signal processing method to compute the data in each discrete frequency channel. Parallel computing can get the whole Brillouin spectrum only in one probe pulse period, so it greatly saves the measurement time, compared with conventional frequency sweeping method.

As each scattering location along the fiber under test returns a Brillouin spectrum, the final measurement result is constructed by Brillouin frequency, power and fiber location, as shown in figure 2. It can be seen from figure 2 that when the temperature and/or strain on the fiber sections changes, correspondingly the Brillouin spectra returning will shift away from the original location. Then by measuring the Brillouin frequency shift, the change of temperature and/or strain will be obtained [4].

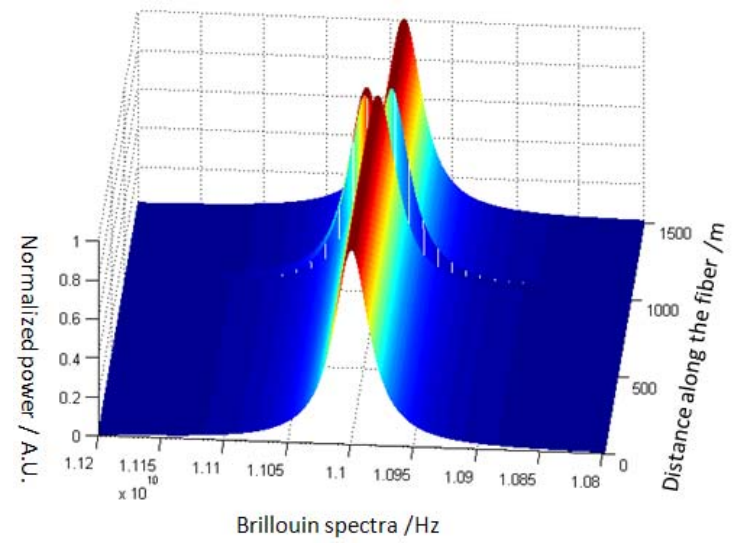

Figure 2. The measurement trace by BOTDR

\section{NEW BOTDR SYSTEM BY PARALLEL COMPUTING}

\section{A. The principle of the new BOTDR system}

Figure 3 shows the schematic diagram of the new BOTDR based on parallel computing scheme. A laser generates light with linewidth less than $1 \mathrm{MHz}$, and through coupler1 the laser light is split into two paths: one is modulated to probe light pulses by an Electro-optic modulator (EOM) and another is used as local oscillator (LO), whose frequency is shifted by about $11 \mathrm{GHz}$ through another EOM. For the probe pulses, the probe pulse period is a litter longer than the round trip time of the probe light for fault location of the scattering in fiber under test. Through a circulator the probe pulses are injected into the fiber under test, then the spontaneous Brillouin scattering spectrum in each location of fiber returns, each having different time sequence. In coupler 2, both the LO and spontaneous Brillouin spectra mix, where they generate new amplitude envelop whose frequency is the difference between them. Then the difference frequency is transferred to electric signals by a photo detector, so the Brillouin electric spectra are obtained.

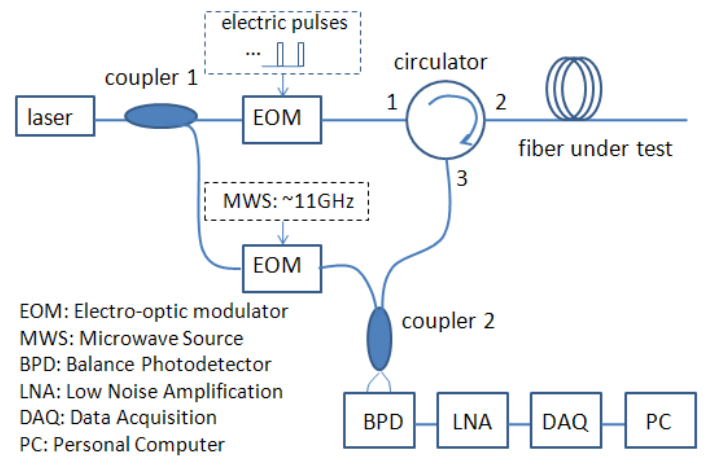

Figure 3. BOTDR based on parallel computing scheme

For extraction of the Brillouin electric spectra, it goes several steps, and this process is shown in figure 4. First, the Brillouin electric spectra are filtered by a band pass filter (BPF) to reduce the noise whose power is much higher than the signals. Then through a low noise amplifier (LNA), the signal power is improved for accurate analog to digital conversion (ADC). Then the digital data are sent to the computer, where parallel digital signal processing is conducted and final results are illustrated.

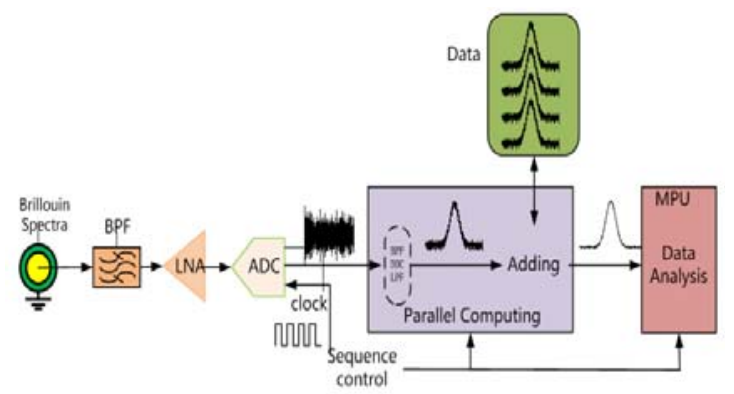

Figure 4. Extraction of Brillouin electric spectrum

For parallel digital signal processing, MATLAB is adopted as program tool for its strong parallel computing capability. The method for parallel computing to extract Brillouin electric spectra is shown in figure 5. From figure 5, it can be seen that the Brillouin electric spectrum can be divided into many discrete frequency channels and each frequency channel corresponds to a band pass filter. Then extraction of the power of each band pass filter can obtain the discrete Brillouin spectrum, as the stems shown in figure 5. Then by digital signal processing method, the power of each frequency channel can be extracted. The process of digital signal processing is as follows [5]: first, by band pass filtering to separate each frequency channel; then by digital down conversion to convert the power of each frequency 
channel to direct current component; at last by digital low pass filtering to extract the power of each frequency channels which forms the discrete Brillouin electric spectra.

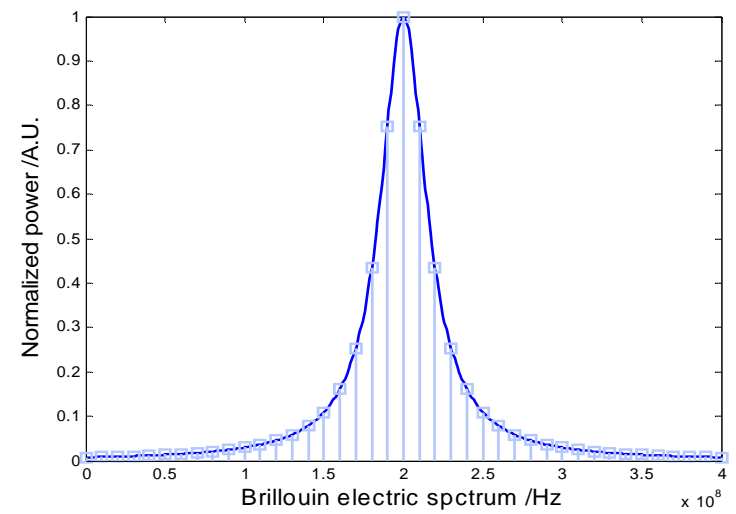

Figure 5. Brillouin electric spectrum obtained by coherent detection method.

\section{B. Results of simulation and discussions}

As scattering location returns a Brillouin spectrum, the measurement results are constructed by each Brillouin spectrum along the fiber under test. For parallel digital signal processing, forty discrete frequency channels with bandwidth of $5 \mathrm{MHz}$ are adopted to separate the Brillouin electric spectra. The frequency range that needs to be separated is set as $400 \mathrm{MHz}$ to make the measurement reach wide temperature and/or strain range. Then, using MATLAB as signal processing tool, we designed forty digital band pass filters, digital down conversion units and digital low pass filters. For parallel computing, the paralleling computing toolbox is also used. The fiber length is set as $10 \mathrm{~km}$ and each scattering cell is $100 \mathrm{~m}$, then the simulation result is shown in figure 6 . It can be seen from figure 6 that each frequency channel corresponds to one trace along the fiber and each scattering location corresponds to a Brillouin spectrum along the frequency axis. By use of parallel computing, the time for signal processing is saved by more than $95 \%$ compared with conventional method.

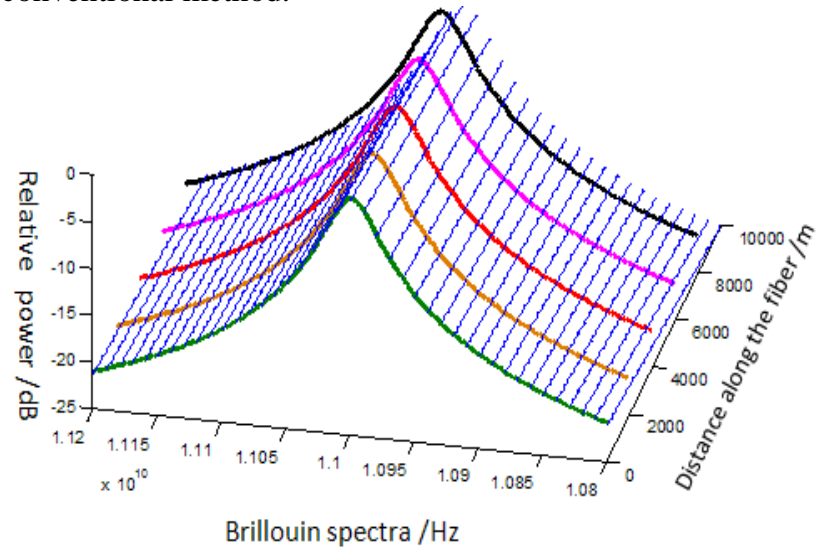

Figure 6. OTDR traces obtained by parallel computing method.
Figure 7 shows the final results that are obtained by fitting the data from figure6. It can be seen from both figure 6 and figure 7 that when the scattering cell on the fiber under test becomes smaller and smaller, the data obtained will be larger and larger , therefore, for conventional frequency sweeping method to extract the Brillouin spectra, much more time will be needed [6]. However, by parallel computing method, if the computing source is satisfied, the measurement time will be greatly saved, which benefits dynamic measurement.

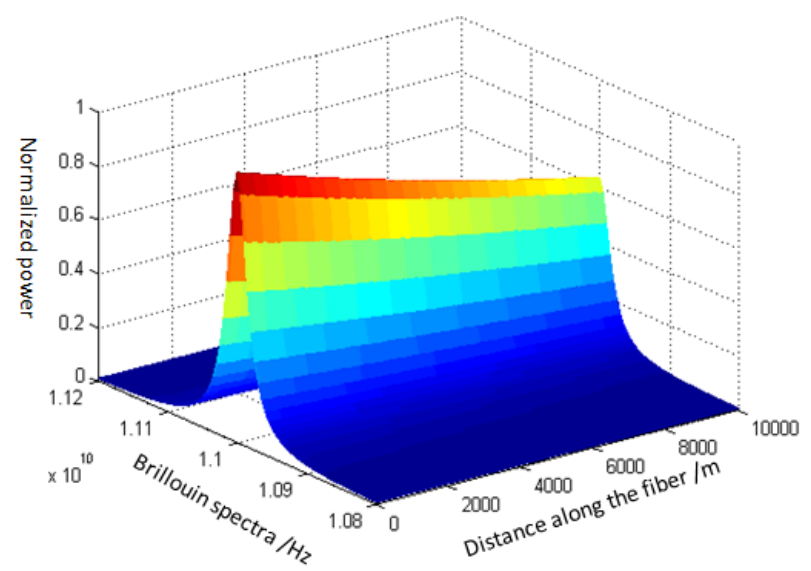

Figure 7. Final OTDR traces obtained by data fitting algorithm.

For distributed optical fiber sensing, paralleling computing is a very important tool that greatly enhances the performance of sensing system. Reference [5] reported paralleling computing method was successfully used in coherent optical time domain reflection for fiber characterization and fault location of optical fiber transmission line, and measurement efficiency was greatly enhanced. For almost all the distributed optical fiber sensing systems, dynamic range, spatial resolution and measurement time are three dominant parameters. At present, pulse coding method is widely used to improve the measurement dynamic range and spatial resolution, but when length and dimension of pulse coding become larger and larger, signal acquisition and processing becomes difficult, especially the far more time will be need to obtain the final measurement results. Therefore, by pulse coding method measurement dynamic range and spatial resolution can be improved, but the dynamic measurement property of sensing systems degrades.

\section{CONCLUSION}

A new signal processing scheme that employs high speed digital signal acquisition and parallel computing is adopted in Brillouin optical time domain reflectometry. In each probe pulse period, the whole Brillouin spectra can be obtained and analyzed. Compared with conventional frequency sweeping method to extract Brillouin spectrum, parallel computing can 
greatly saves the measurement time which benefit dynamic measurement.

\section{ACKNOWLEDGMENT}

The authors would like to thank Dr. Fan Zhu for his help on signal processing program design.

\section{REFERENCES}

[1] T. Horiguchi, K. Shimizu, T. Kurashima, M. Tateda, and Y. Koyamada, "Development of a Distributed Sensing Technique Using Brillouin Scattering,” J. Lightwave Technol., vol. 13, pp. 1296-1302, July 1995.

[2] K. Shimizu, T. Horiguchi, Y. Koyamada, and T. Kurashima, "Coherent Self-Heterodyne Brillouin OTDR for measurement of
Brillouin Frequency Shift Distribution in Optical Fibers," J. Lightwave Technol., vol. 12, pp. 730-736, May 1994.

[3] T. Kurashima, T. Horiguchi, and M. Tateda, "Thermal Effects of Brillouin Gain Spectra in Single Mode Fibers," IEEE Photon. Technol. Lett., vol. 2, pp. 718-720, October 1990.

[4] K. Shimizu, T. Horiguchi, Y. Koyamada, and T. Kurashima, "Coherent Self-Heterodyne Detection of Spontaneously BrillouinScattered Light Waves in a Single-Mode Fiber,” Opt. Lett., vol.18, pp. 185-187, February 1993.

[5] Lidong Lu, Yuejiang Song, Fan Zhu, and Xuping Zhang, “Coherent optical time domain reflectometry using three frequency multiplexing probe," Optics and Lasers in Engineering, vol. 50, pp. 1735-1739, December 2012.

[6] M. Soto, G. Bolognini, and F. Pasquale, "Analysis of Pulsu Modulated Fromat in Coded BOTDA Sensors," Opt. Expr., vol. 18, pp. 1489-1503, June 2010. 